\title{
Broadband CMOS Gilbert Down-Converter Utilizing a Low-Loss Step- Impedance Rat-Race Coupler
}

\author{
Hung-Ju Wei, Chinchun Meng, and Sheng-Wen Yu
}

Department of Communication Engineering, National Chiao Tung University, Hsinchu, 300 Taiwan, R.O.C.

\begin{abstract}
A Broadband Gilbert Down-Converter with stepimpedance rat-race coupler is demonstrated using $0.13 \mu \mathrm{m}$ standard CMOS technology. The proposed method utilizes higher effective dielectric-constant, phase-inverter and step-impedance techniques simultaneously to make the rat-race coupler more compact $(\sim 64 \%$ reduction) and phase-balanced over a wide operating bandwidth. The down-converter has NF of $16 \mathrm{~dB}$ and LO-to-RF isolation of better than $35 \mathrm{~dB}$. The mixer core consumes $2.9 \mathrm{~mA}$ at $\boldsymbol{V}_{d d}=1.8 \mathrm{~V}$.
\end{abstract}

Index Terms - Rat-race coupler, phase inverter, step impedance, and Gilbert mixer.

\section{INTRODUCTION}

The conventional hybrid-ring directional coupler [1] has the balanced output only at a certain frequency. In order to improve bandwidth, the methods of opposing short-circuit end and twisting-connection form the embryo of phase inverter [2][3]. Then, the design of arm-impedance, the technique of slow-wave and the usage of lumped elements are studied [4-6] to achieve arbitrary power-division ratio or size-reduction.

As the speed of CMOS technology increases by device scaling in the past decades, a new silicon micro-wave and millimeter-wave IC era has finally begun. Because quarterwave-length becomes shorter at higher frequency, more and more passive components, such as $3-\mathrm{dB} 90^{\circ}$ and $0^{\circ} / 180^{\circ}$ hybrid couplers, can be fully-integrated on the silicon-based circuits. However, the first issue is to overcome the loss from the low resistivity $(\sim 10 \Omega \cdot \mathrm{cm})$ silicon substrate. To alleviate the problem, the technologies of high-resistivity substrate, micromachining, silicon-on-insulator (SOI), ground shields and floating shielding [7] are proposed and discussed [8]. As described in [9], E-band ring-hybrid couplers above backsideground are implemented in the silicon technology. Because of low dielectric constant, the length of each arm is up to $300 \mu \mathrm{m}$ and $320 \mu \mathrm{m}$ for $60 \mathrm{GHz}$ and $77 \mathrm{GHz}$ couplers.

In this paper, a Gilbert down-converter, using a miniature step-impedance rat-race coupler directly on the lossy silicon substrate, has NF of $16 \mathrm{~dB}$ and LO-to-RF isolation of better than $35 \mathrm{~dB}$ over a wide bandwidth. The arm length of $10 \mathrm{GHz}$ and $15 \mathrm{GHz}$ rat-race couplers are $860 \mu \mathrm{m}$ and $630 \mu \mathrm{m}$, respectively. If compared with E-band ring-hybrid couplers, this work only needs a half of equivalent length for each side. The proposed method utilizes higher silicon dielectric constant, phase-inverter and step-impedance structures to make the rat- race coupler more compact and easier to be integrated in the silicon circuits for millimeter-wave applications.

\section{CIRCUIT DESIGN}

Referring to Fig. 1(a), the phase inverter, twisting the signal (S) and ground (G) paths of coplanar strip-line (CPS) of ratrace coupler, not only takes the place of the $3 / 4$ wavelength section for size-reduction, but also provides a differential signal between port-2 and port-4 for any operating frequency. From our derived equation (1) for the lossy condition, the matching points will be gradually separated if lower characteristic impedance $\left(Z_{C P S}<\sqrt{2} Z_{0}\right)$ is chosen, so as to enlarge the matching bandwidth. Under lossless condition ( $\alpha=0), \hat{z}$ becomes the well-known relation of $\sqrt{2\left(1-\cot ^{2} \theta\right)} \cdot \hat{z}$ is the normalized characteristic impedance of the transmission line, with respect to the terminal impedance, $Z_{0}$.

$$
\hat{z}=\sqrt{2+2 \operatorname{coth}^{2}(\gamma \ell)}=\sqrt{2\left[1+\operatorname{coth}^{2}(\alpha+j \beta) \ell\right]} .
$$

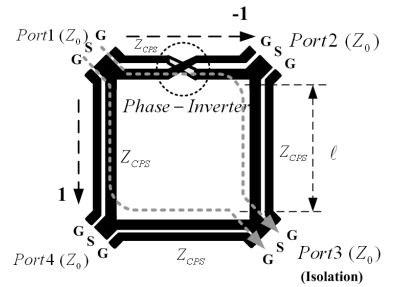

(a)

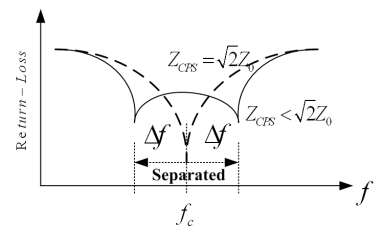

(b)

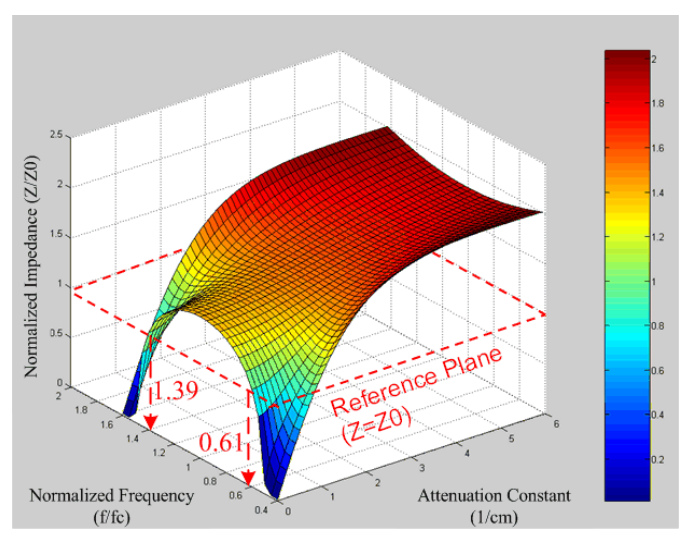

(c)

Fig. 1. Principle of a rat-race coupler with phase-inverter on the loss condition. 
Figure 1(c) shows that the normalized impedance is a function of the normalized frequency and attenuation constant. The characteristic impedance of the transmission lines, $Z$, is chosen to be $50 \Omega$, and $f_{C}$ is the center frequency of each arm of a rat-race coupler. If $\alpha=0\left(\mathrm{~cm}^{-1}\right)$, the bandwidth ratio of $f_{\max } /\left.f_{\min }\right|_{\alpha=0}$ is equal to $2.27\left(f_{\max } / f_{c}=1.39\right.$ and $\left.f_{\min } / f_{c}=0.61\right)$. When $\alpha$ increases up to $0.8\left(\mathrm{~cm}^{-1}\right)$, the bandwidth ratio of $f_{\max } /\left.f_{\text {min }}\right|_{\alpha=0.8}$ is extended to 2.7. Under the specified impedance, the loss condition is a benefit to the bandwidth of the coupler return-loss. However, the insertion loss must be considered in the design procedure.

Because the operating mode of CPS is analogous to that of the coplanar waveguide (CPW), the signal transmitted in the CPS is almost carried between the adjacent metals. The desired impedance of $Z_{C P S}$ can be designed by choosing the width and space of the metals. Relative to the transmission line with grand plane, CPS directly on silicon substrate has shorter effective wavelength due to higher effective dielectric constant. The shorter a transmission line is, the lower the substrate effect is. In other words, the degradation of performance caused by lossy substrate can be mitigated.

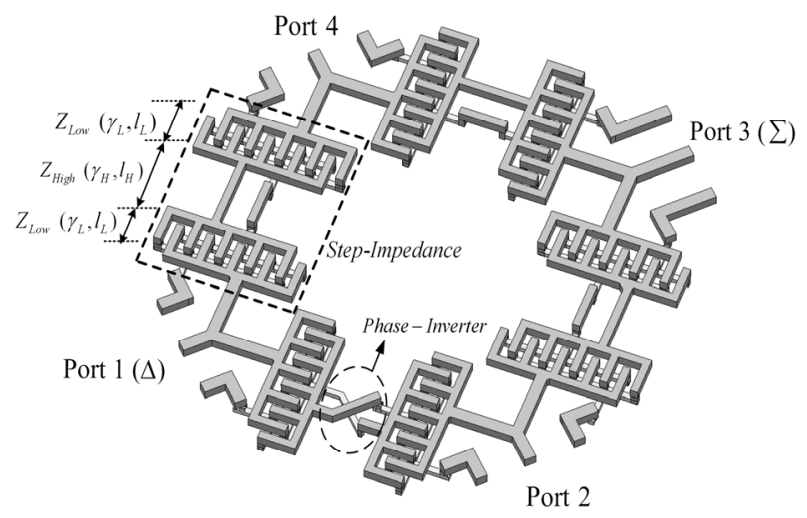

Fig. 2. 3D view of the step-impedance rat-race coupler.

$$
\begin{aligned}
& {\left[\begin{array}{cc}
\cosh \left(\gamma_{L} \ell_{L}\right) & \hat{z}_{\text {low }} \sinh \left(\gamma_{L} \ell_{L}\right) \\
\frac{1}{\hat{z}_{\text {low }}} \sinh \left(\gamma_{L} \ell_{L}\right) & \cosh \left(\gamma_{L} \ell_{L}\right)
\end{array}\right]\left[\begin{array}{cc}
\cosh \left(\gamma_{H} \ell_{H}\right) & \hat{z}_{\text {High }} \sinh \left(\gamma_{H} \ell_{H}\right) \\
\frac{1}{\hat{z}_{\text {High }}} \sinh \left(\gamma_{H} \ell_{H}\right) & \cosh \left(\gamma_{H} \ell_{H}\right)
\end{array}\right]\left[\begin{array}{cc}
\cosh \left(\gamma_{L} \ell_{L}\right) & \hat{z}_{\text {low }} \sinh \left(\gamma_{L} \ell_{L}\right) \\
\frac{1}{\hat{z}_{\text {low }}} \sinh \left(\gamma_{L} \ell_{L}\right) & \cosh \left(\gamma_{L} \ell_{L}\right)
\end{array}\right]} \\
& =\left[\begin{array}{cc}
\cosh (\gamma \ell) & \hat{z} \sinh (\gamma \ell) \\
\frac{1}{\hat{\lambda}} \sinh (\gamma \ell) & \cosh (\gamma \ell)
\end{array}\right]
\end{aligned}
$$

In order to shrink the length of each arm further, the method of step-impedance is utilized. As shown in Fig. 2, the stepimpedance structure consists of a higher-impedance $\left(Z_{H i g h}\right)$ section and two lower-impedance $\left(Z_{\text {Low }}\right)$ sections. The ABCD matrix of the step-impedance section for the lossy condition is described in (2). As the ratio of $Z_{\text {High }} / Z_{\text {Low }}$ increases, the shorter total length $\left(2 l_{L}+l_{H}\right)$ can be achieved. The low-highlow impedance is viewed as the capacitive-inductivecapacitive section. Because the minimum space is limited by the process, the low-impedance section is designed as an interdigital capacitor (IDC).

Figure 3 shows a $10 \mathrm{GHz}$ step-impedance rat-race coupler fabricated in a standard CMOS process. The length of each arm in the conventional rat-race coupler is up to $1340 \mu \mathrm{m}$. After using step-impedance techniques, the length is reduced to only $860 \mu \mathrm{m}$ and $64 \%$ size-reduction is achieved. The $3-\mathrm{dB}$ bandwidth is from $5 \mathrm{GHz}$ to $15 \mathrm{GHz}$, and the insertion loss is about 6 7 dB. Compared with [10], the improvement of insertion loss means that problem of loss is mitigated. At the same time, the phase between port- 2 and port- 4 is under the differential condition $\left(180^{\circ}\right)$ for much wider frequency range. The maximum phase and magnitude errors for the operation frequencies of $5 \sim 15 \mathrm{GHz}$ are within 4 degrees and $1 \mathrm{~dB}$, respectively. The isolation between delta and sum port, $\left|\mathrm{S}_{31}\right|$, is always better than $30 \mathrm{~dB}$. The measured results reveal that a phase-inverter rat-race coupler with the step-impedance miniature method directly on silicon substrate becomes feasible for balanced RF circuits.

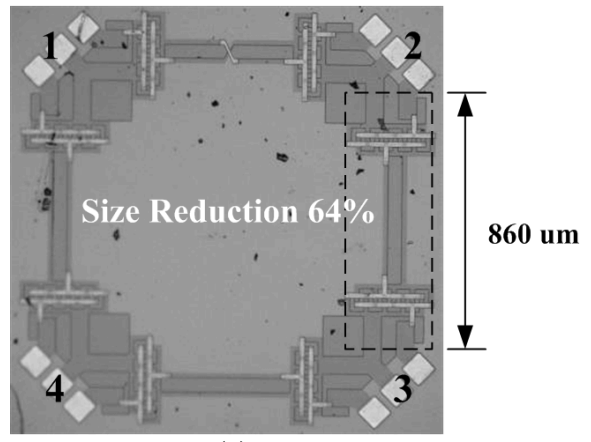

(a)

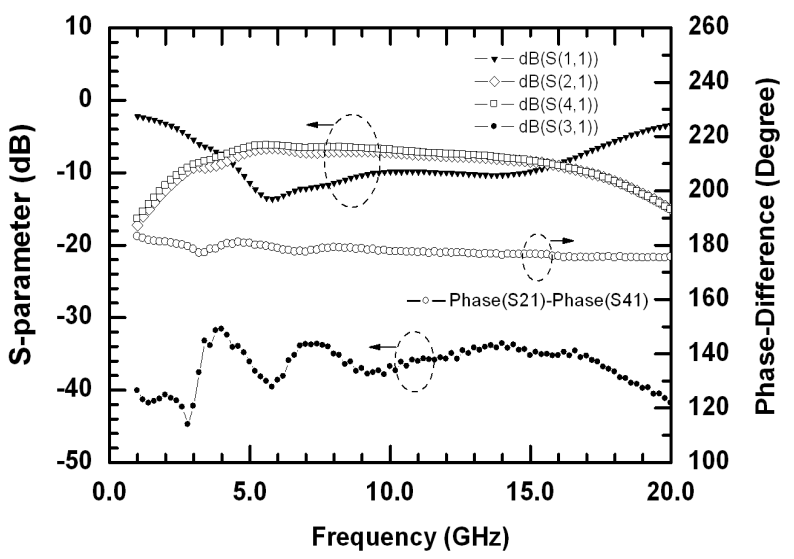

(b)

Fig. 3. (a) The chip micrograph, (b) measured S-parameter and phase of the step-impedance rat-race coupler.

Figure 4 shows a practical application of the step-impedance rat-race coupler in the Gilbert down-conversion mixer. The step-impedance rat-race coupler is employed in the RF stage to generate the wideband differential signal, and followed by the common-gate-configured transistors, $M_{1} \sim M_{2}$, which have superior frequency response and provide impedance matching for the required impedance, $50 \Omega$. The device size is ten- 
finger with unit gate-width of $2.5 \mu \mathrm{m}$. The length of each arm of the rat-race coupler at $f_{\text {center }}=15 \mathrm{GHz}$ is further shrunk to $630 \mu \mathrm{m}$. A thick metal-8 of $3.3 \mu \mathrm{m}$ is used to reduce the metal loss. At LO port, a Marchand balun generates an out-ofphase signal to drive the Gilbert mixer core.

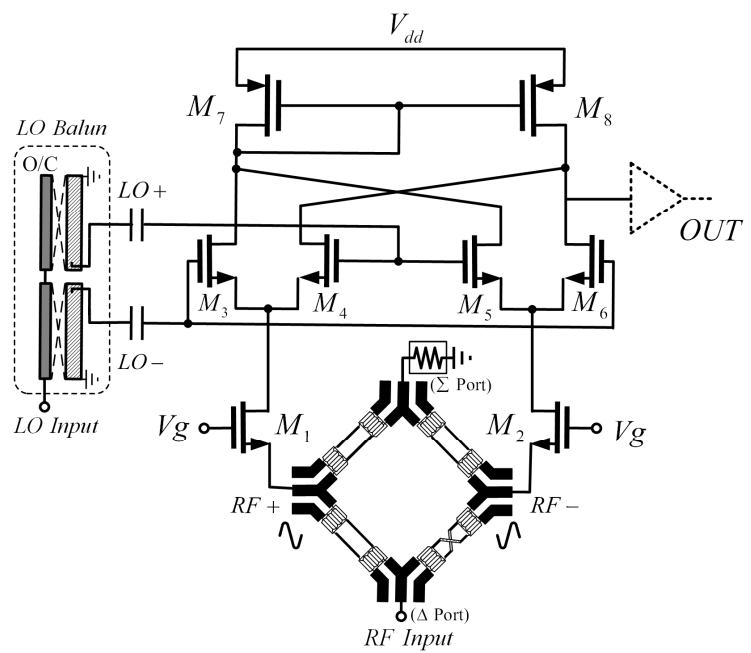

Fig. 4. Simplified circuit diagram of the down-converter using a stepimpedance rat-race coupler at the RF input stage.

\section{EXPERIMENTAL RESULTS}

The broadband down-converter using a step-impedance ratrace coupler is implemented using $0.13 \mu \mathrm{m}$ standard CMOS technology as shown in Fig. 3. The chip size is $1.4 \times 1.8 \mathrm{~mm}^{2}$, and the mixer core consumes $2.9 \mathrm{~mA}$ at $V_{d d}=1.8 \mathrm{~V}$.

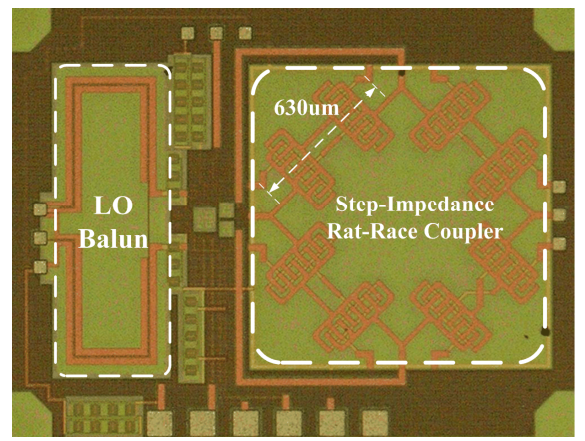

Fig. 5. Chip micrographs of the down-converter using a stepimpedance rat-race coupler. $\left(1.4 \times 1.8 \mathrm{~mm}^{2}\right)$

Figure 6 shows the conversion gain and LO-to-RF isolation. When measured at $\mathrm{IF}=100 \mathrm{MHz}$, the conversion gain is above $8 \mathrm{~dB}$ from $7 \mathrm{GHz}$ to $18 \mathrm{GHz}$. The measured LOto-RF isolation is below than $35 \mathrm{~dB}$ all over the operating range. Figure 7 shows the conversion gain and noise figure versus IF frequency under $f_{R F_{-} \text {input }}=9.1 \mathrm{GHz}$. The minimum NF of $16.2 \mathrm{~dB}$ indicates that the step-impedance rat-race coupler in the first stage successfully converts the received power to common-gate input, and suffers less from substrate-loss. As shown in Fig. 8, the measured $\mathrm{P}_{1 \mathrm{~dB}}$ and $\mathrm{IIP}_{3}$ are around -12 $\mathrm{dBm}$ and $-2 \mathrm{dBm}$, respectively.

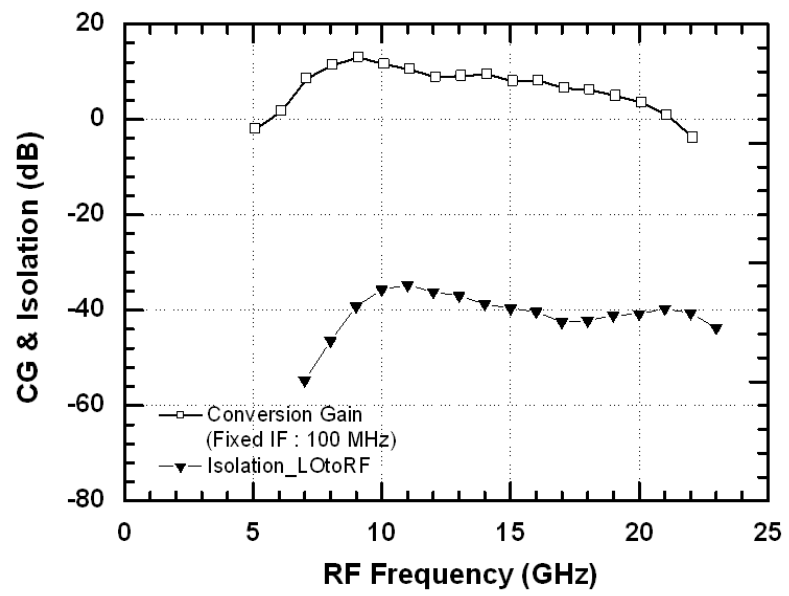

Fig. 6. Measured conversion gain and LO-to-RF isolation verse RF Frequency.

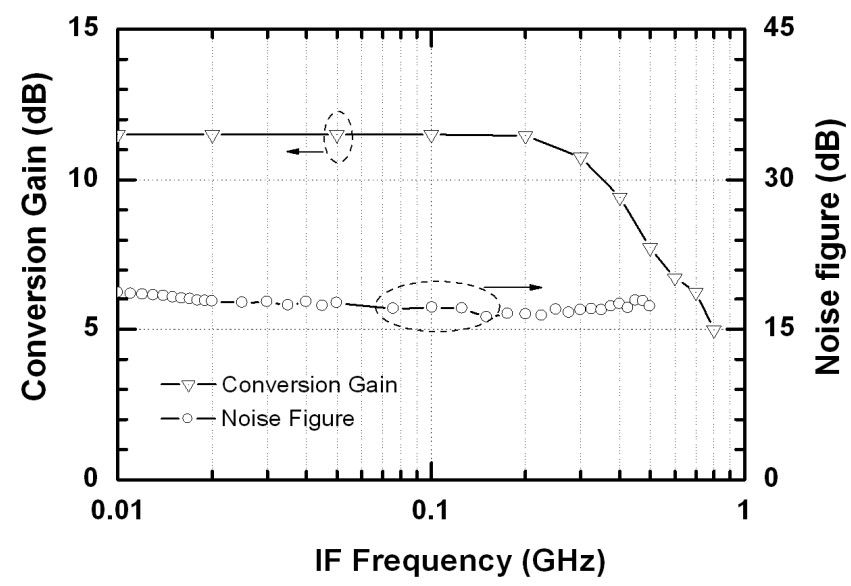

Fig. 7. Measured conversion gain and noise figure verse IF Frequency.

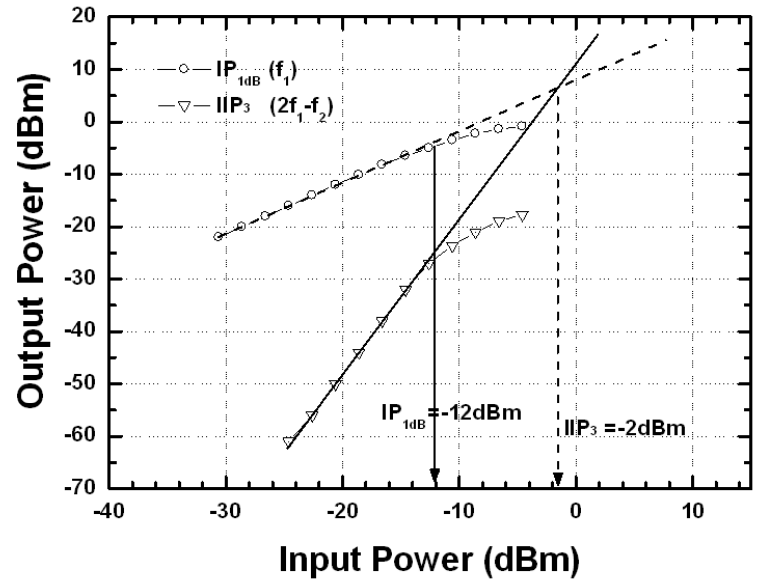

Fig. 8. Measured power performances, $\mathrm{IP}_{3 \mathrm{~dB}}$ and $\mathrm{IIP}_{3}$. 


\section{CONCLUSION}

This paper describes the design and performance of a broadband Gilbert down-converter with the step-impedance rat-race coupler using $0.13 \mu \mathrm{m}$ standard CMOS technology. The methods of higher effective dielectric-constant, phase inverter and step-impedance are simultaneously utilized to shrink the size of the rat-race coupler about $64 \%$ and to generate an out-of-phase signal over operating bandwidth. The down-converter, using the proposed rate-race coupler at the RF input port, has NF of $16 \mathrm{~dB}$ and LO-to-RF isolation of better than $35 \mathrm{~dB}$. The core circuit consumes $2.9 \mathrm{~mA}$ at $V_{d d}$ $=1.8 \mathrm{~V}$. From the measured noise figure, the rat-race coupler suffers from substrate loss slightly. The shorter a transmission-line is, the lower the substrate-effect is.

\section{ACKNOWLEDGEMENT}

This work is supported by National Science Council of Taiwan, Republic of China under contract numbers NSC 952221-E-009-043-MY3, NSC 97-2221-E-009-171 and NSC 982218-E-009-008-MY3, by the Ministry of Economic Affairs of Taiwan under contract number 96-EC-17-A-05-S1-020, and by MoE ATU Program under contract number 95W803. The authors would like to thank National Chip Implementation Center (CIC) for technical support.

\section{REFERENCES}

[1] C. Y. Pon, "Hybrid-Ring Directional Coupler for arbitrary power division," IEEE Trans. Microw. Theory Tech., vol. 9, no. 6, pp. 529-535, Nov. 1961.

[2] S. March, "A wideband stripline hybrid ring," IEEE Trans. Microw. Theory Tech., vol. 16, no. 6, pp. 361, Nov. 1968.
[3] S. Rehnmark, "Wide-band balanced line microwave hybrids," IEEE Trans. Microw. Theory Tech., vol. 25, no. 10, pp. 825-830, Oct. 1977.

[4] A. K. Agrawal and G. F. Mikucki, "A printed circuit hybrid-ring directional coupler for arbritrary power divisions," in IEEE MTT-S Int. Microwave Symp. Dig., 1986, pp. 139-142.

[5] S. J. Parisi, " $180^{\circ}$ lumped element hybrid," in IEEE MTTS Int. Microw. Symp. Dig., Jun. 13-15, 1989, pp. 12431246.

[6] K. Wu, and T. Wang, "Size-Reduction and BandBroadening Design Technique of Uniplanar Hybrid Ring Coupler Using Phase Inverter for $\mathrm{M}(\mathrm{H}) \mathrm{MIC}$ 's," IEEE Trans. Microw. Theory Tech., vol. 47, no. 2, pp. 198-206, Feb. 1999.

[7] T. S. D. Cheung and J. R. Long, "Shielded passive devices for silicon-based monolithic microwave and millimeter-wave integrated circuits," IEEE J. Solid-State Circuits, vol. 41, no. 5, pp. 1183-1200, May 2006.

[8] C. H. Doan, S. Emami, A. M. Niknejad, and R. W. Brodersen, "Millimeter-wave CMOS design," IEEE J. Solid-State Circuits, vol. 40, no. 1, pp. 144-155, Jan. 2005.

[9] M. K. Chirala and B. A. Floyd, "Millimeter-wave Lange and ring hybrid couplers in a silicon technology for Eband applications," in IEEE MTT-S Int. Microw. Symp. Dig., Jun. 11-16, 2006, pp. 1547-1550.

[10] S. C. Tseng, C. C. Meng, C. H. Chang, S. H. Chang, and G. W. Huang. "A Silicon Monolithic Phase-Inverter RatRace Coupler Using Spiral Coplanar Striplines and Its Application in a Broadband Gilbert Mixer," IEEE Trans. Microw. Theory Tech., vol. 56, no. 8, pp. 1879-1888, Aug. 2008. 